\title{
A COMPARATIVE STUDY TO EVALUATE THE EFFECT OF DIFFERENT IRRIGATING SOLUTIONS ON THE SMEAR LAYER
}

\author{
Hayam Y. Hassan * and Ahmed M. Negm**
}

\begin{abstract}
Aim: This study compared the capability of Biopuer MTAD, QMix ${ }^{\mathrm{TM}} 2 \mathrm{in} 1,17 \%$ EDTA, 6.25\% Morinda Citrifolia juice, 2\% Nano-Chitosan, 2\% Chitosan, 2\% Chlorhexidine and distilled water in smear layer removal.

Methods: eighty extracted premolars having single root canals were distributed into eight groups allocated to the final irrigant used. Roots were separated in bacco-lingual direction into two equal halves then analyzed using Scanning Electron Microscope (SEM) at the coronal, middle, and apical parts.

Results: In the coronal and middle parts of the roots the Biopure MTAD showed the highest mean values of smear layer elimination, followed by $17 \%$ EDTA, QMix ${ }^{\mathrm{TM}} 2 \mathrm{in} 1$, and then $6.25 \%$ MCJ. While 2\% nano-chitosan, $2 \%$ chitosan, $2 \%$ CHX and distilled water showed the least mean values of smear layer elimination. The capability of smear layer elimination decreased in the apical third for Biopuer MTAD, 17\% EDTA, QMix ${ }^{\mathrm{TM}} 2$ in 1 and $6.25 \%$ MCJ. It was only inhibited with $2 \%$ nano-chitosan, $2 \%$ chitosan, $2 \% \mathrm{CHX}$ and distilled water.
\end{abstract}

Conclusion: the study concluded that Biopuer MTAD had the highest mean value for smear layer removal in middle, coronal and to a lesser degree in apical thirds.

KEYWORDS: Smear layer, Biopuer MTAD, QMix ${ }^{\mathrm{TM}}$ 2in1, EDTA, Chitosan.

\section{INTRODUCTION}

Root canal therapy includes the use of instruments in combination with irrigating solutions in order to eliminate bacteria from lumen of the pulp. Although instrumentation reduces the number of microorganisms ${ }^{(1)}$, it generates a layer of organic and inorganic residues covering the root canal known as smear layer. The smear layer is a rough coating containing pulp tissue, odontoblastic process, necrotic debris, microorganisms ${ }^{(2)}$. Despite the controversy over keeping or maintaining the smear layer, it has been revealed that the smear layer might be infected and keep the bacteria inside the dentinal tubules. This layer has also been shown to prevent the diffusion of intracanal medications and irrigants into dentinal tubules ${ }^{(3)}$.

* Assistant Professor of Endodontics, Faculty of Dentistry, Suez Canal University, Ismailia, Egypt

** Assistant Professor of Endodontics, Faculty of Dentistry, Ahram Canadian University, Giza, Egypt. 
Sodium hypochlorite $(\mathrm{NaOCl})$ followed by EDTA has an effect in eliminating pulpal tissue remnants and components of smear layer ${ }^{(4)}$. Biopure MTAD shown promise action in smear layer elimination ${ }^{(5)}$. However, the antimicrobial efficacy and substantivity of this irrigant combination has been challenged ${ }^{(6)}$. Biopure MTAD is also relatively ineffective against $E$. faecalis biofilms ${ }^{(7)}$ which are more resistant to antimicrobial medications. It is effective in eliminating canal wall smear layers but demineralizes intraradicular dentin ${ }^{(8)}$. QMix ${ }^{\mathrm{TM}} 2 \mathrm{in} 1$ irrigating solution is also a mixture of endodontic irrigant containing EDTA, chlorhexidine gluconate, and a non-specified detergent that might give the action of $17 \%$ EDTA $^{(9)}$.

The main drawbacks, safety property, and ineffectiveness of most of the synthetic irrigating solutions to mainly clean root canals advise that neith ofer of these irrigating solutions are perfect. At this time, few natural fruit juices that might be used complementary to $\mathrm{NaOCl}$ or Chlorhexidine (CHX) as applicable irrigants. Morinda Citrifolia juice (MCJ) has therapeutic and antimicrobial properties ${ }^{(10)}$, suggesting that it is effective to be used as an endodontic irrigant.

The limitations of current disinfectants have prompted researchers to look for new alternatives. Chitosan, is a nontoxic cationic natural biopolymer that is obtained through the alkaline deacetylation process of chitin. This biopolymer possesses activity against many fungi and bacteria ${ }^{(11)}$. Antibacterial nanoparticles, like nano-chitosan, have a significant antibacterial activity when compared with other antibacterial powders. This is due to a wide surface area and charge density that enables it to react with the negative charge surface of bacterial cell walls, lead to bacterial cell death ${ }^{(12)}$.

This study compared the capability of Biopuer MTAD, QMix ${ }^{\mathrm{TM}} 2$ in1, 17\% EDTA, 6.25\% Morinda Citrifolia juice, $2 \%$ nano-chitosan, $2 \%$ Chitosan, $2 \%$ Chlorhexidine and distilled water for smear layer removal.

\section{MATERIALS AND METHODS}

Ethical clearance was taken before starting the study. Eighty extracted human premolars with single root canal were collected. Teeth with cracks or fracture lines were eliminated after examining using loops. The teeth were cleansed and kept in saline solution.

The working length was adjusted to $17 \mathrm{~mm}$ using a diamond disk (D\&Z, Darmstadt, Germany). The root canals were accessed and the initial standardized crown down technique for coronal preparation was done with Gates-Glidden drills (Dentsply Maillefer, Ballaigues, Switzerland) up to size 4. Sequentially K files (Dentsply-Maillefer, Ballaigues, Switzerland) were used up to size 40 for apical preparation with $2.5 \% \mathrm{NaOCl}$ irrigation (Oxford Laboratory, Mumbai, India), followed by washing with $5 \mathrm{ml}$ of saline. The samples were distributed into eight groups $(n=10)$ matching to the final irrigation used.

- Group 1: Biopure MTAD (Dentsply Tulsa Dental Specialties, Tulsa, OK).

- Group 2: QMix ${ }^{\mathrm{TM}} 2 \mathrm{in} 1$ (Dentsply Tulsa Dental Specialties, Oklahoma, USA).

- Group 3: 17\% EDTA (ethylene diamine tetracetic acid) (Prevest Denpro Limited Company Jammu, India).

- Group 4: 6.25\% Morinda Citrifolia (NONI) Juice (MCJ) (Dynamic Health Laboratories, Inc. Brooklyn, USA).

- Group 5: 2\% Nano-Chitosan (Nano Tech, Dream Land, Egypt).

- Group 6: 2\% Chitosan (Nano Tech, Dream Land, Egypt).

- Group 7: 2\% Chlorhexidine gluconate (CHX) (El Nasr Pharmaceutical Chemicals Company, Egypt).

- Group 8: Distilled water (control). 
Chitosan was prepared by deacetylation of the chitin, which forms the exoskeleton of crustaceans. Dissolving the high molecular weight chitosan powder in $1 \%$ acetic acid solution to form a $2 \%$ chitosan solution. For preparation of $2 \%$ nanochitosan, add $2 \%$ chitosan solution to thirty percent of $\mathrm{H}_{2} \mathrm{O}_{2}$ (hydrogen peroxide) aqueous solution in the ratio of $1: 100$. The reaction was done at $60^{\circ} \mathrm{C}$, regulating the solution to $\mathrm{pH} 8$. Formed solution was centrifuged at $\mathrm{pH} 7$ using deionized water. The formed paste was freezed, thawed at $60^{\circ} \mathrm{C}$. Then, milled the chitosan in TJH-2-4L multidimensional swipe nano-ball-mill with a driving motor of $7 \mathrm{~kW}$. $1 \%$ acetic acid was added to nano-chitosan, then stirred for $2 \mathrm{~h}$ using a magnetic stirrer till crystalline homogeneous solutions with $3.2 \mathrm{pH}$ formed ${ }^{(13,14)}$.

For group 1: each root canal was irrigated with freshly prepared Biopure MTAD according to manufacturer instructions, $1 \mathrm{ml}$ as an initial rinse and soaked for 5 minutes. A final wash was done using $4 \mathrm{ml}$ for each canal. Finally, each root canal was washed by $5 \mathrm{ml}$ of distilled water and dried with sterile paper points.

For the other groups from 2 to 8 , root canals were irrigated with $5 \mathrm{ml}$ for 2 minutes of each freshly prepared and standardized irrigant corresponding to each group according to manufactures instructions. Irrigants were delivered into the root canals with Max-I-Probe, a side vented endodontic irrigating needle (Dentsply-Rinn, Elgin,IL) until the working length using manual technique. Finally, each root canal was washed with $5 \mathrm{ml}$ of distilled water and dried with sterile paper points.

All the root canals were sectioned in the buccolingual direction using diamond discs to make deep grooves on the external root surfaces. Then the grooves were split using chisel and mallet.

The specimens were dehydrated by ethyl alcohol then mounted on coded stubs, air dried, placed in a vacuum and coated with a 300 A gold coat for one half of each root. The specimens were then examined and photographed using Scanning Electron Microscope (SEM) (JEOL JXA-810, Technics Co., Tokyo, Japan). The dentine surfaces were observed and photographed at the coronal, middle, and apical parts at X 1000 magnification. The presence or absence of smear layer and visualization of the dentinal tubules. The scores were directed to the rating system established by Madison and Hokett $1997^{(15)}$

0- No, no removal of smear layer, no dentinal tubules visible.

1- Partial, partial removal of smear layer, some dentinal tubules visible.

2. Complete, complete removal of smear layer, all dentinal tubules visible.

The data obtained were statistically analyzed.

\section{RESULTS}

Kruskal Wallis analysis of variance was used for intragroup comparison. The mean rank values recorded that there was a significant difference among the tested irrigants in smear layer elimination in the coronal, middle and apical thirds $(\mathrm{P}<0.05)$. For the coronal and middle thirds of the roots, Biopure MTAD showed the highest mean value of smear layer elimination, followed by $17 \%$ EDTA then $\mathrm{QMix}^{\mathrm{TM}} 2 \mathrm{in} 1$, (they were almost equal) followed by, $6.25 \%$ MCJ. While $2 \%$ nano-chitosan, $2 \%$ chitosan, $2 \% \mathrm{CHX}$ and distilled water showed the least mean values of smear layer elimination. The capability of smear layer elimination decreased in the apical third with Biopure MTAD, 17\% EDTA, QMix ${ }^{\mathrm{TM}} 2 \mathrm{in} 1$ and $6.25 \%$ MCJ. It was inhibited for $2 \%$ nano-chitosan, $2 \%$ chitosan, $2 \% \mathrm{CHX}$ and distilled water, as shown in (Table 1) and Figs. (1-8) 
TABLE (1) Mean Rank values of smear layer removal at the coronal, middle and apical thirds of all the tested solutions.

\begin{tabular}{|l|c|c|c|}
\hline \multirow{2}{*}{ Tested solutions } & \multicolumn{3}{|c|}{ Mean Rank $^{1}$} \\
\cline { 2 - 4 } & Coronal & Middle & 66.35 \\
\hline Biopure MTAD & 65.00 & 66.15 & 64.80 \\
\hline QMix ${ }^{\text {TM}}$ 2in1 & 59.80 & 64.30 & 64.80 \\
\hline $17 \%$ EDTA & 62.40 & 64.30 & 28.05 \\
\hline $6.25 \%$ MCJ & 36.85 & 30.25 & 25.00 \\
\hline $2 \%$ Nano-Chitosan & 31.80 & 24.75 & 25.00 \\
\hline $2 \%$ Chitosan & 31.80 & 24.75 & 25.00 \\
\hline $2 \%$ Chlorohexidine Gluconate (CHX) & 21.85 & 27.50 & 25.00 \\
\hline Distilled Water (Control) & 14.50 & 22.00 & 72.869 \\
\hline Chi-Square value & 55.512 & 66.297 & 0.0001 \\
\hline P value & 0.0001 & 0.0001 & \\
\hline
\end{tabular}

${ }^{1}$ The Kruskal Wallis test (one-way analysis of variance by ranks), $(P<0.05)$.

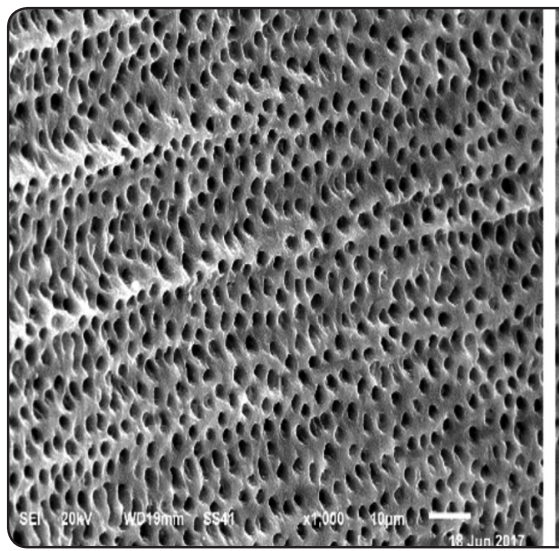

a) Coronal

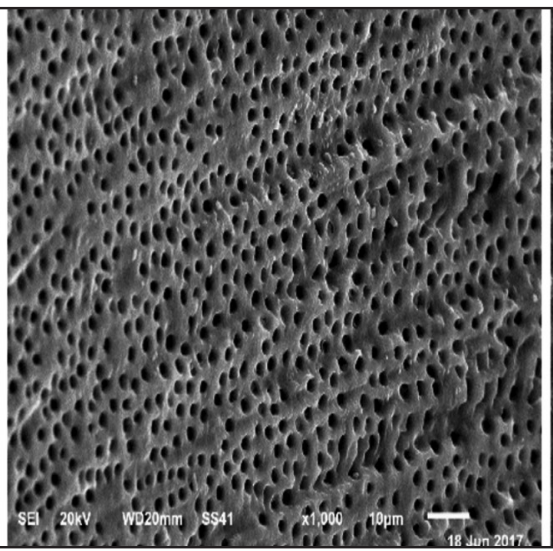

b) Middle

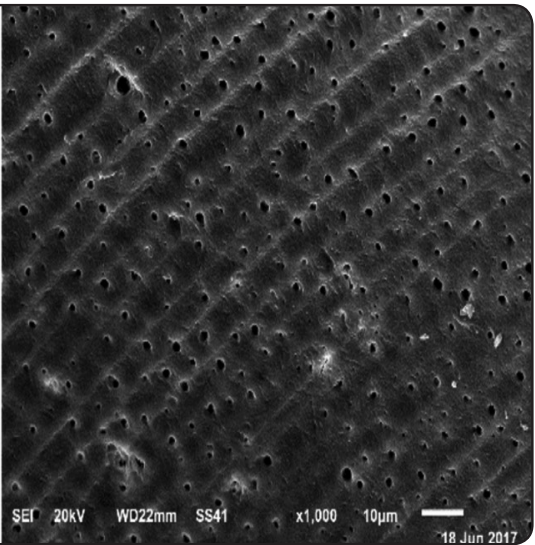

c) Apical

Fig. (1) Scanning electron microscope (SEM) images of Biopure MTAD (Group 1) at the (a) coronal, (b) middle and (c) apical thirds (original magnification $1000 \times$ ). 


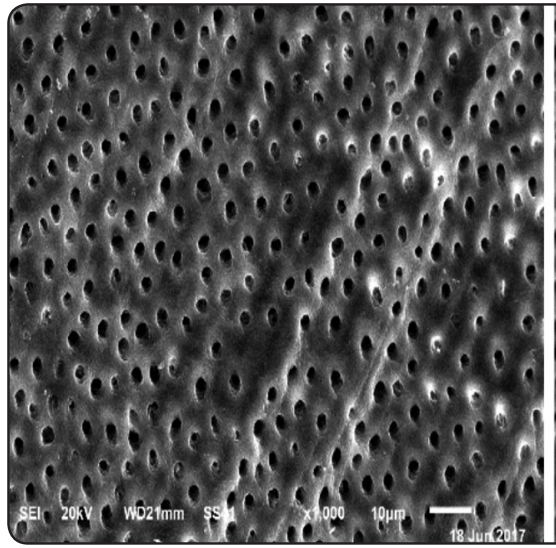

a) Coronal

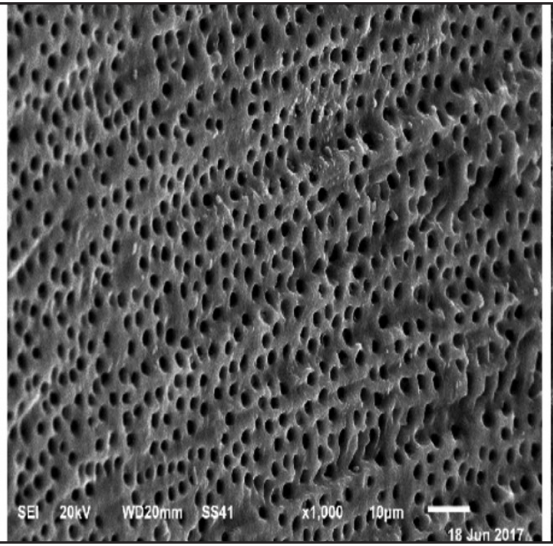

b) Middle

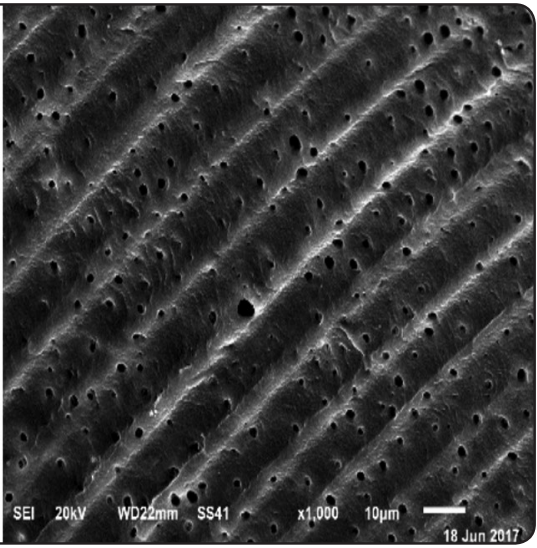

c) Apical

Fig. (2) Scanning electron microscope (SEM) images of QMixTM2in1 (Group 2) at the (a) coronal, (b) middle and (c) apical thirds (original magnification $1000 \times$ ).

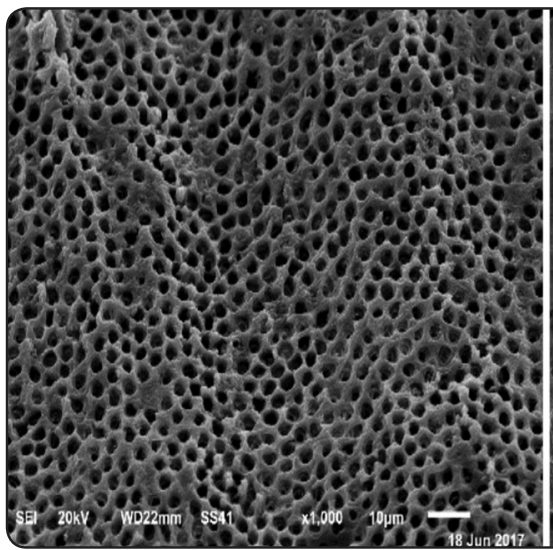

a) Coronal

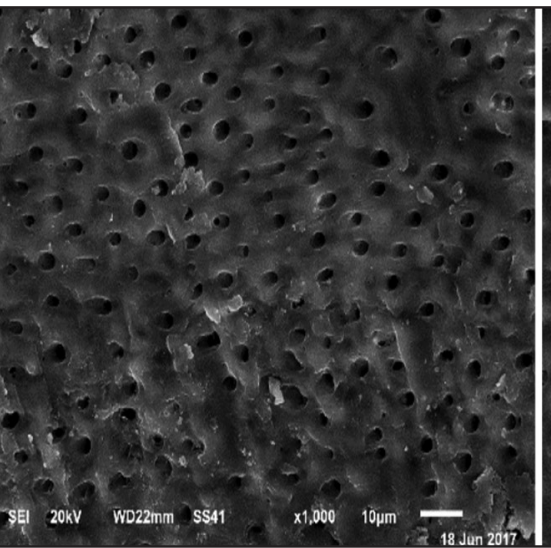

b) Middle

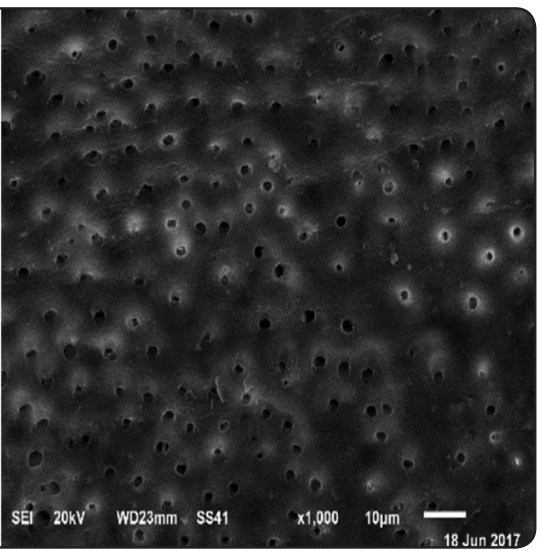

c) Apical

Fig. (3) Scanning electron microscope (SEM) images of 17\% EDTA (Group 3) at the (a) coronal, (b) middle and (c) apical thirds (original magnification $1000 \times$ ).

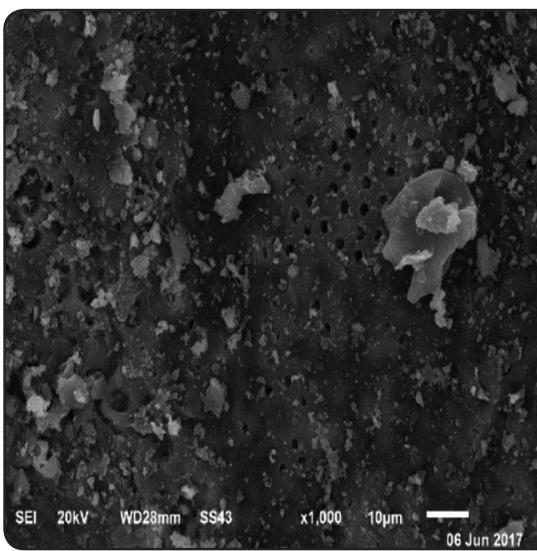

a) Coronal

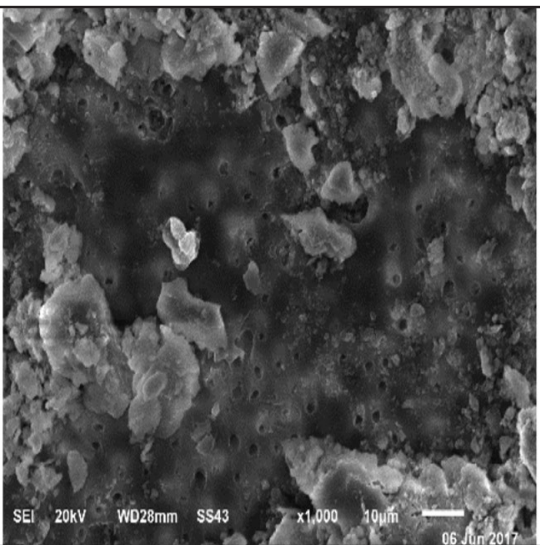

b) Middle

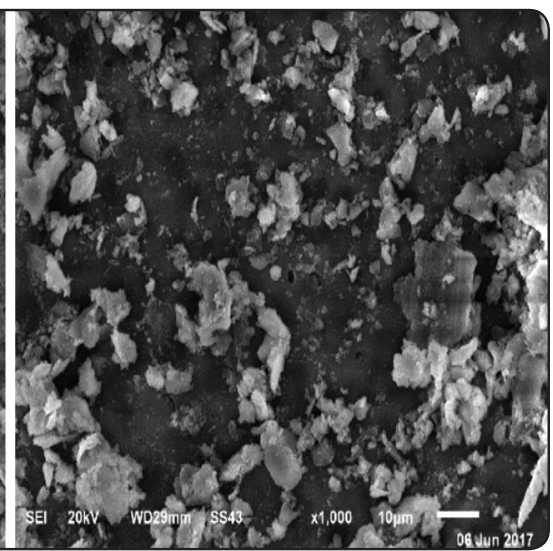

c) Apical

Fig. (4) Scanning electron microscope (SEM) images of 6.25\% Morinda citrifolia Juice (MCJ) (Group 4) at the (a) coronal, (b) middle and (c) apical thirds (original magnification 1000x). 


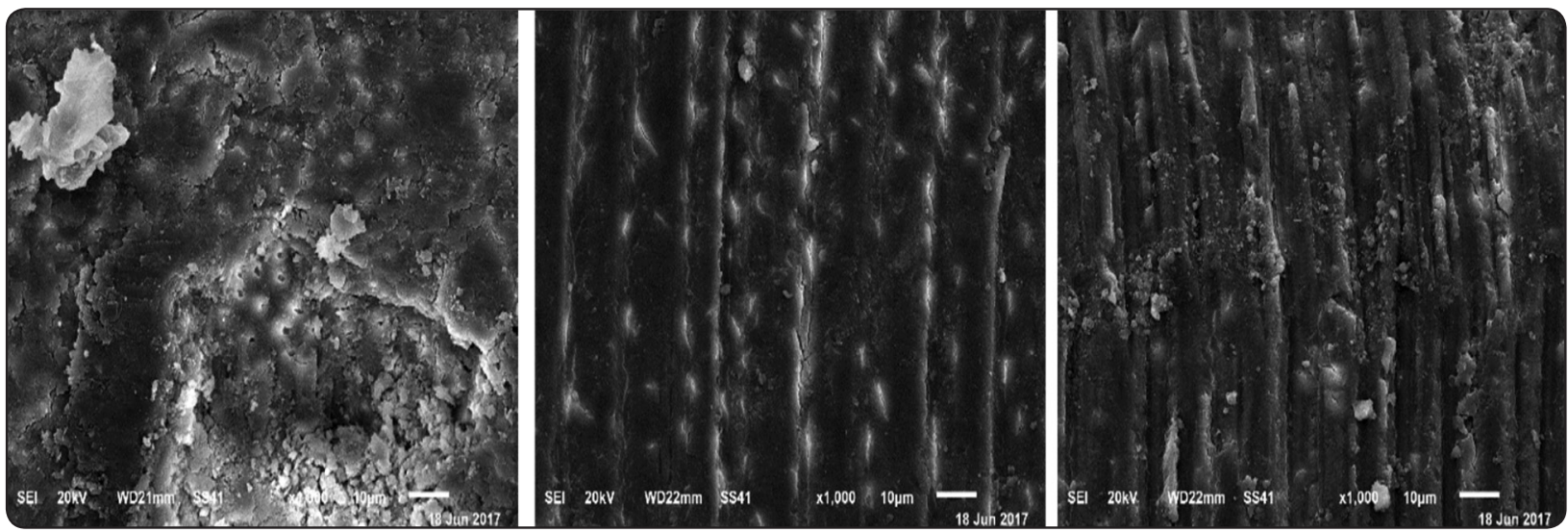

a) Coronal

b) Middle

c) Apical

Fig. (5) Scanning electron microscope (SEM) images of 2\% Nano-Chitosan (Group 5) at the (a) coronal, (b) middle and (c) apical thirds (original magnification 1000x).

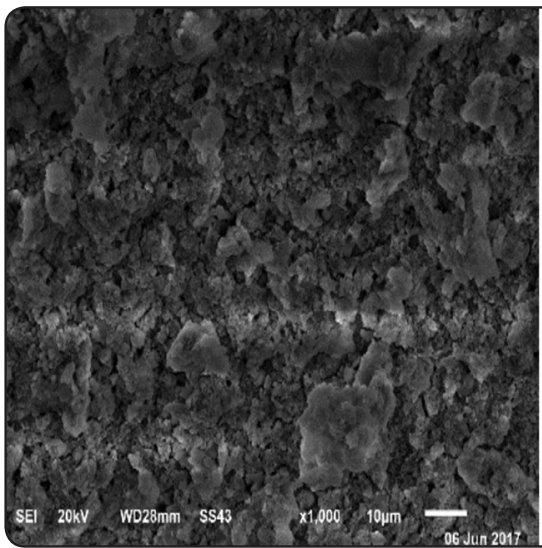

a) Coronal

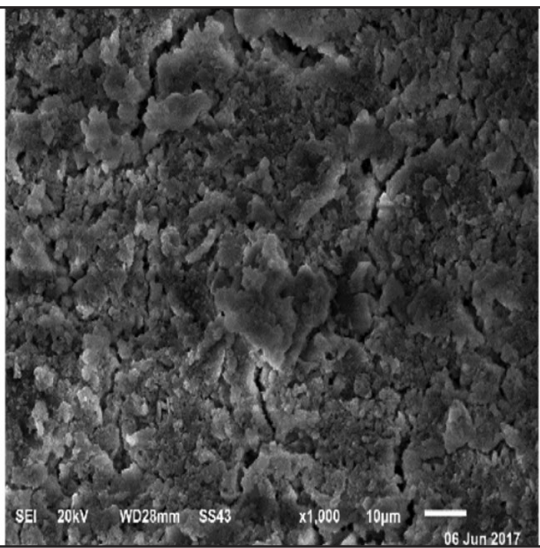

b) Middle

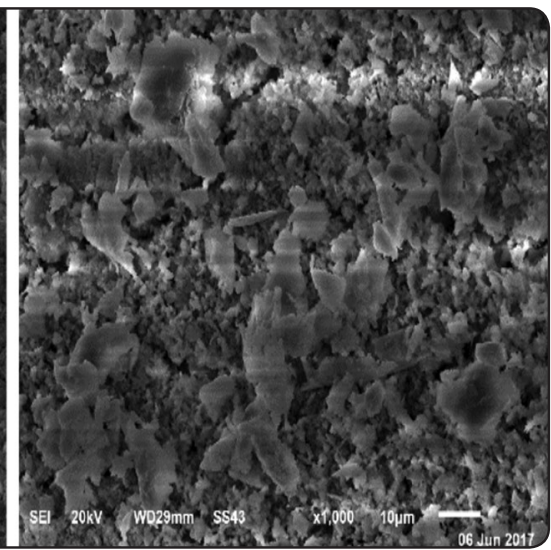

c) Apical

Fig. (6) Scanning electron microscope (SEM) images of 2\% Chitosan (Group 6) at the (a) coronal, (b) middle and (c) apical thirds (original magnification $1000 \times$ ).

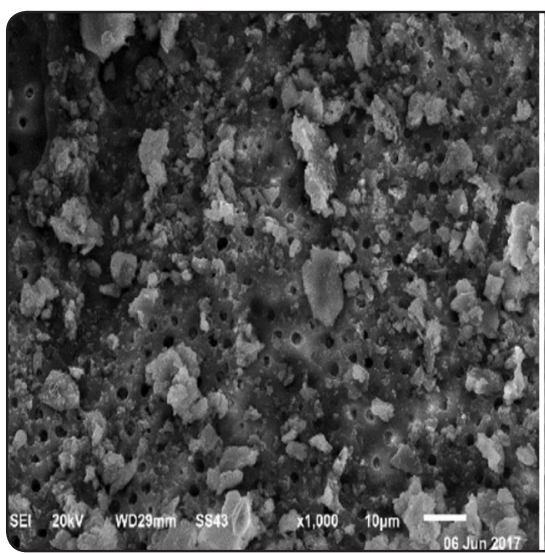

a) Coronal

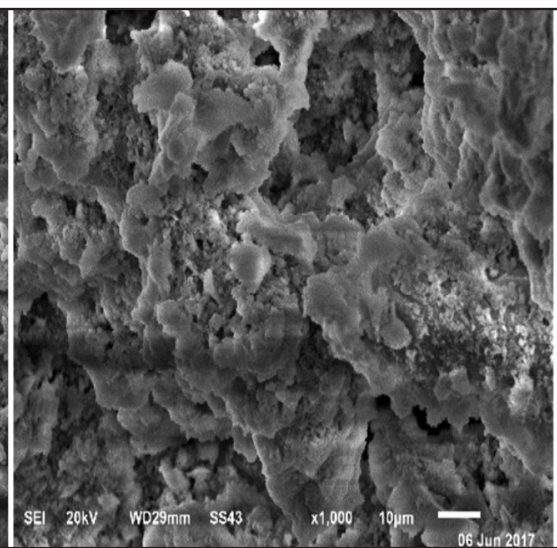

b) Middle

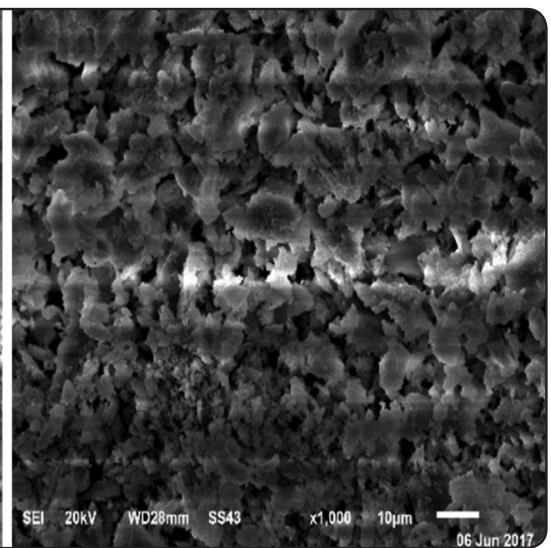

c) Apical

Fig. (7) Scanning electron microscope (SEM) images of 2\% Chlorohexidine gluconate (CHX) (Group 7) at the (a) coronal, (b) middle and (c) apical thirds (original magnification 1000x). 


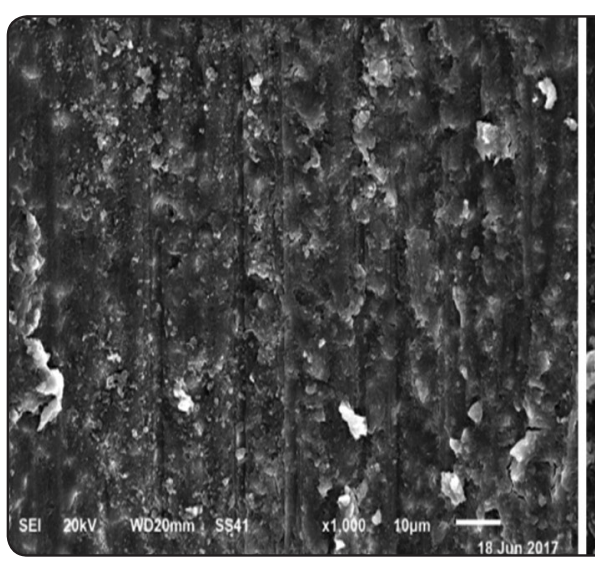

a) Coronal

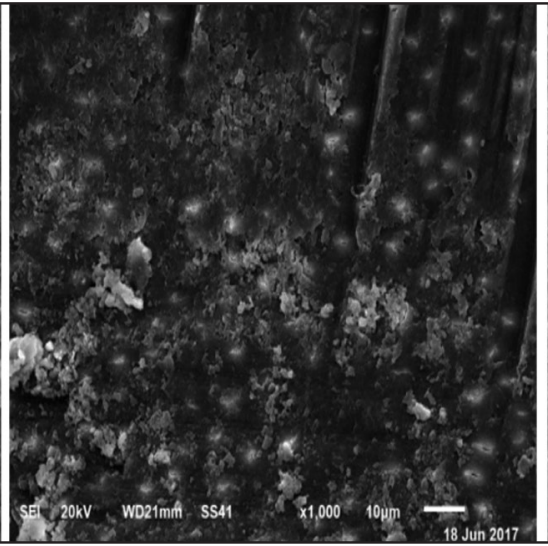

b) Middle

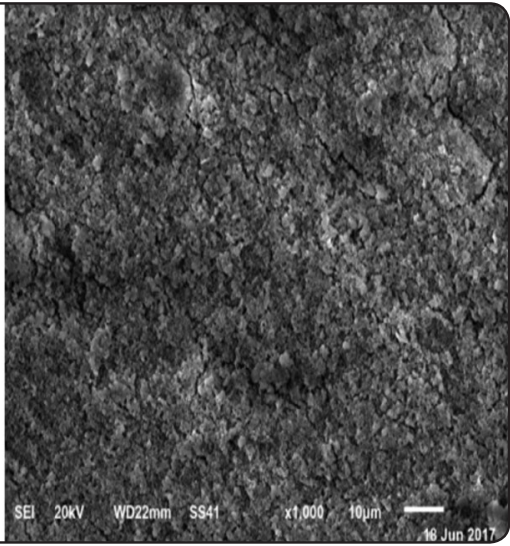

c) Apical

Fig. (8) Scanning electron microscope (SEM) images of Distilled Water (Control) (Group 8) at the (a) coronal, (b) middle and (c) apical thirds (original magnification 1000×).

\section{DISCUSSION}

The efficiency of any irrigating solution to eliminate smear layers at different zones of the root canal depends on the type of the irrigant and the delivery method ${ }^{(16)}$. Method of delivery was not examined at this study that evaluated the effectiveness of irrigant solutions rather than irrigation.

The current study presented efficient elimination of smear layer in coronal and middle parts rather than apical parts of the root canals. This came in agreement with several studies that showed efficient cleaning of coronal and middle parts of the canals even when using different irrigation volumes and several times ${ }^{(17-20)}$. Coronal and middle thirds have wider canal diameter that exposes dentin to a greater volume of irrigants, giving better flow of the solution, and hence increasing the smear layer elimination capacity ${ }^{(18,20)}$.

Biopuer MTAD showed the highest mean value of smear layer elimination due to the active ingredient of $4.25 \%$ citric acid and detergent polysorbate 80 that decreased the surface tension leading to increased permeability allowing Biopuer MTAD to permeate better enhancing the decalcification effect (21) These came in an agreement with De-Deus et al ${ }^{(8)}$ Torabinejad et al ${ }^{(22)}$ and Ghoddusi et al ${ }^{(23)}$ who concluded that Biopuer MTAD demineralizes dentin faster than 17\% EDTA without affecting the structural integrity of dentinal tubules.

EDTA and QMix $^{\mathrm{TM}} 2$ in1 showed almost equal values for smear layer elimination due to the fact that the active ingredient in both irrigants is EDTA. This came in agreement with Dai et al ${ }^{(24)}$ who found that EDTA has the same effect as two versions of QMix $^{\text {TM }}$ 2in1. EDTA possess high surface tension than Biopuer MTAD which reduce its ability to wet root canal surface, also the hydrogen exchange of calcium from dentin resulting in a subsequent lowering in $\mathrm{pH}$. Therefore the capability of EDTA reduced by time ${ }^{(25)}$.

Findings showed that MCJ had significantly reduced ability to remove smear layer which came in agreement with Murray et al ${ }^{(26)}$ who found that although MCJ had strong antibacterial action and did not have an effect on smear layer elimination. To enhance its effect, a final rinse with EDTA 17\% was done.

Among the premises of the study Chitosan and Nano-Chitosan have shown reduction in smear layer but with less mean value than the previously mentioned irrigants as mentioned by Geethapriya 
et $\mathbf{a l}^{(27)}$ who tested the effect of Chitosan accompanied by EDTA as an efficient smear layer removal with less erosion in dentinal tubules.

Based on the results of this investigation, $\mathrm{CHX}$ had no effect on smear layer completely and left most of dentinal tubules occluded. This was in harmony with studies which showed that CHX has strong antibacterial effect with no effect on the dentine components of root canal ${ }^{(28-30)}$.

Nearly, all the groups revealed less or no effect on smear layer at the apical part of the root canal. This is due to the flow and backflow capability of the fluid which are less at this part due to the narrow diameter and the increase in depth of the root canal, together with the lesser amount of dentinal tubules than coronally and abundance of sclerotic dentine ${ }^{(31)}$.

\section{CONCLUSION}

The present study concluded that Biopuer MTAD had the highest mean value for smear layer removal in middle, coronal and to a minimal effect in apical parts while the rest of irrigants performed well in coronal and middle parts with no effect apically.

\section{REFERENCES}

1- Bystrom A and Sundqvist G: Bacteriologic evaluation of mechanical root canal instrumentation in endodontic therapy. Scand J Dent Res. 89: 321-8, 1981.

2- Torabinejad M, Handysides R, Khademi A and Baklan LK: Clinical implications of the semar layer in endodontics: a review. Oral Surg Oral Med Oral Pathol Oral Radiol Endod, 94: 658-6, 2002.

3- Ostravik D and Haapasalo M: Disinfection by endodontic irrigant and dressings of experimentally infected dentinal tubules. Endod Dent Traumatol, 6: 142-9, 1990.

4- Baumgartner JC and Mader CL: A scanning electron microscopic evaluation of four root canal irrigation regimens. J Endod. 13: 147-57, 1987.

5- Davis JM, Maki J and Bahcall JK: An in vitro comparison of the antimicrobial effects of various endodontic medi- caments on Enterococcus faecalis. J Endod. 33: 567-9, 2007.

6- Kho P and Baumgartner JC: A comparison of the antimicrobial efficacy of $\mathrm{NaOCl} / \mathrm{Biopure} \mathrm{MTAD}$ versus $\mathrm{NaOCl} /$ EDTA against Enterococcus faecalis. J Endod. 32: 652-5, 2006.

7- Clegg MS, Vertucci, FJ and Walker C: The effect of exposure to irrigant solutions on apical dentin biofilms in vitro. J Endod. 32: 434-7, 2006.

8- De-Deus G, Reis C and Fidel S: Dentin demineralization when subjected to Biopure MTAD: a longitudinal and quantitative assessment. J Endod. 33: 1364-8, 2007.

9- Dai L, Khechen K and Khan S: The effect of QMixTM2in1, an experimental antibacterial root canal irrigant, on removal of canal wall smear layer and debris. J Endod. 37: 80-4, 2011.

10- Wang MY, West BJ and Jensen CJ: Morinda citrifolia (Noni): a literature review and recent advances in Noni research. Acta Pharmacol Sin. 23: 1127-41, 2002.

11- Del Carpio-Perochena A, Kishen A and Shrestha A: Antibacterial properties associated with chitosan nanoparticle treatment on root dentin and 2 types of endodontic sealers. J Endod. 41: 1353-8, 2015.

12- Shi Z, Neoh KG and Kang ET: Antibacterial and mechanical properties of bone cement impregnated with chitosan nanoparticles. Biomaterials. 27: 2440-9, 2006.

13- Ambore SM, Kanthale S, Mukesh G, Chandrakant R, and Avinash D: A brief overview on chitosan applications. Indo American Journal of Pharm Research. 3:1563-76, 2013.

14- Yadav P, Chaudhary S, Saxena R, Talwar S, and Yadav S: Evaluation of antimicrobial and antifungal efficacy of chitosan as endodontic irrigant against Enterococcus Faecalis and Candida Albicans biofilm formed on tooth substrate. J Clin Exp Dent. 9:361-7, 2017.

15- Madison JG and Hokett SD: The effect of different tetracyclines on the root surface of instrumented, periodontally involved human teeth: a comparative scanning electron microscopic study. J Periodontol. 68:739-45, 1997.

16- Gu, LS, Kim JR and Ling: Review of contemporary irrigant agitation techniques and devices.J Endod. 35: 791804, 2009.

17- Baumgartner JC and Mader CL: A scanning electron microscopic evaluation of four root canal irrigation regimens. J Endod. 13: 147-57, 1987. 
18- Torabinejad M, Khademi AA and Babagoli J: A new solution for the removal of the smear layer. J Endod. 29: 170$5,2003$.

19- Abbott PV, Heijkoop PS, Cardaci SC, Hume WR and Heithersay GS: A SEM study of the effects of different irrigation sequences and ultrasonic. Int Endod J. 24: 30816,1991 .

20- Teixeira CS, Felippe MC, and Felippe WT: The effects of application time of EDTA and $\mathrm{NaOCl}$ on intracanal smear layer removal: a SEM analysis. Int Endod J. 38: 285-90, 2005.

21- Giardino L, Ambu E and Becce C: Surface tension comparison of four common root canal irrigants and two new irrigants containing antibiotic. J Endod. 32:1091-3, 2006.

22- Torabinejad M, Cho Y, Khademi AA, Bakland LK and Shabahang S: The effect of various concentrations of sodium hypochlorite on the ability of Biopuer MTAD to remove the smear layer. J Endod. 29:233-9, 2003.

23- Ghoddusi J, Rohani A and Rashed T: An evaluation of microbial leakage after using Biopuer MTAD as a final irrigation. J Endod. 33:173-6, 2007.

24- Dai L, Khechen K, Khan S, Gillen B, Loushine BA, Wimmer CE, Gutmann JL, Pashley D and Tay FR: The effect of $\mathrm{QMix}^{\mathrm{TM}} 2 \mathrm{in} 1$, an experimental antibacterial root canal irrigant, on removal of canal wall smear layer and debris. $\mathrm{J}$ Endod. 37: 80-4, 2011.
25- Hulsmann M, Heckendorff M, and Lennon A: Chelating agents in root canal treatment: mode of action and indications for their use. Int Endod J. 36:810-30. 2003.

26- Murray PE, Farber RM, Namerow KN, Kuttler S and Garcia-Godoy F: Evaluation of Morinda Citrifolia as an endodontic irrigant. J Endod. 34:66-70, 2008.

27- Geethapriya N, Subbiya A and Sukumaran VG: Effect of chitosan-ethylenediamine tetraacetic acid on Enterococcus faecalis dentinal biofilm and smear layer removal. J Conserv. 9: 472-7, 2016.

28- Yamashita JC, Tanomaru M and Leonardo MR: Scanning electron microscopic study of the cleaning ability of chlorhexidine as a root canal irrigant. Int Endod J. 36:3914. 2003.

29- Okino LA, Siqueira EL and Santos M: Dissolution of pulp tissue by aqueous solution of chlorhexidine digluconate and chlorhexidine digluconate gel. Int Endod J.31:38-41, 2004 .

30- Saghiri MA, and Delvarani P: A study of the relation between erosion and microhardness of root canal dentin. Oral Surg Oral Med Oral Pathol Oral Radiol Endod. 108:29-34, 2009 .

31- Vasiliadis, L, Darling, AI and Levers BG: The amount and distribution of sclerotic human root dentin. Arch Oral Biol. 28: 645-9, 1983. 